Ann. Zootech., I973, 22 (I), I27-I3I.

NOTE

\title{
ESSAI DE GROUPAGE DES AGNELAGES APRÈS UNE LUTTE NATURELLE, CHEZ LA BREBIS
}

\author{
M.-J. BOSC \\ avec la collaboration technique de P. Petrequin* et B. Mirman* \\ Station de Recherches sur la Physiologie de la Reproduction, \\ Centre de Recherches de Tours, I. N. R. A., \\ Nouzilly, 37380 Monnaie \\ * Station de Recherches sur la Physiologie de la Lactation, \\ Domaine expérimental de Brouessy, \\ Magny-les-Hameaux \\ 78470 Saint Rémy les Chevreuse \\ RÉSUMÉ
}

Un essai de groupage des agnelages, après une lutte naturelle est décrit pour un troupeau de brebis de race Préalpes.

La propriété de la dexaméthasone d'induire la parturition a été utilisée à cet effet. Tous les 4 ou 5 jours, les brebis qui atteignaient le début de la période d'agnelage (soit 2 à 6 jours avant le terme moyen), ont reçu une injection intramusculaire de $16 \mathrm{mg}$ de ce produit. Pour chacun des groupes d'animaux ainsi constitués, les mise-bas se sont produites en majorité (9o p. Ioo environ) le $2^{\mathbf{e}}$ et le $3^{\mathrm{e}}$ jour qui ont suivi le traitement.

Cette expérience montre que l'on peut mieux organiser la surveillance du troupeau et qu'on obtient une réduction appréciable des jours avec agnelages.

En fin de gestation, chez la Brebis, l'injection d'une dose suffisante de dexaméthasone induit la parturition en un temps déterminé (Bosc, I970). Comme aucune conséquence fâcheuse ne suit la mise-bas ainsi provoquée chez la Brebis (Bosc, 1970-1972), contrairement à la Vache (Adams, I 969) et à la Chèvre (Bosc, non publié) pour lesquelles il n'y a pas expulsion du placenta, une technique de groupage des agnelages a été proposée lorsque les brebis ont été préalablement synchronisées pour l'ovulation et l'insémination. Afin d'étudier la possibilité de grouper les mise-bas au cas où les brebis sont soumises à une lutte naturelle, nous avons entrepris l'expérience dont nous rapportons ici les résultats. 


\section{CONDITIONS EXPÉRIMENTALES}

224 brebis, de race Préalpes, d'un seul troupeau, saillies entre le I 7 août et le I er octobre I97I, ont été utilisées. Elles ont été soumises à une «lutte en main " c'est-à-dire à une saillie naturelle contrôlée de façon très stricte.

Elles ont reçu une injection intramusculaire de $16 \mathrm{mg}$ de dexaméthasone (solution de phosphate de fluoro-méthyl prednisolone), vers i $8 \mathrm{~h}$, en choisissant le jour de traitement de façon que l'efficacité du traitement d'induction de la parturition soit bonne et que le risque d'agnelage naturel soit faible. Comme nous l'avons précédemment montré (Bosc, I972). cette efficacité diminue lorsqu'on s'écarte de la période d'agnelage qui s'étend du $\mathrm{I}_{4} 4^{\mathrm{e}}$ au $\mathrm{I}_{5} 2^{\mathrm{e}}$ jour de gestation pour les brebis considérées. Le risque d'agnelage naturel dépend de la probabilité d'agnelage ce jour-là et les jours suivants.

Cette probabilité est indiquée dans le tableau suivant; elle a été calculée à partir des données obtenues sur 50 animaux témoins du même troupeau dont la durée de gestation moyenne était égale à $I_{4} 8,3 \hat{j}$ (-t: $I, 95 \mathrm{j}$ ), en supposant que les durées de gestation suivaient une loi de répartition normale;

\begin{tabular}{|c|c|c|c|c|c|c|c|c|c|}
\hline $\begin{array}{c}\text { Jours } \\
\text { de gestation }\end{array}$ & $\mathrm{I} 44^{\mathrm{e}}$ & $145^{\mathrm{e}}$ & $1_{4} 6^{e}$ & $147^{\mathrm{e}}$ & $14^{8}$ & $I+9^{e}$ & I $50^{\mathrm{e}}$ & $\mathrm{I} 5 \mathrm{I}^{\mathrm{e}}$ & ${ }^{1} 5^{2^{e}}$ \\
\hline $\begin{array}{l}\text { Probabilité } \\
\text { d'agnelage }\end{array}$ & 0,03 & 0,07 & $0, \mathbf{I} 3$ & 0,18 & 0,20 & 0,17 & $\mathrm{O}, \mathrm{I} \mathbf{I}$ & 0,06 & 0,02 \\
\hline
\end{tabular}

Les mises en lots des animaux ont donc été faites suivant cette méthode, en traitant le même jour le plus grand nombre de brebis se trouvant au $\mathrm{I} 43^{\mathrm{e}}, \mathrm{I} 44^{\mathrm{e}}$ et $\mathrm{r} 45^{\mathrm{e}}$ jour de gestation. Connai sant le nombre de brebis qui sont au $\mathrm{I}_{4} 2^{\mathrm{e}}$ jour de gestation ce jour-là, on peut estimer le risque d'agnelage pour les jours suivants : si ce risque est important, les brebis sont alors traitées; s'il est faible, elles sont reportées au lot suivant et traitées au I $_{4} 6$ e jour de gestation.

Une surveillance quotidienne des brebis a été assurée entre $5 \mathrm{~h}$ et $23 \mathrm{~h}$, l'heure des agnelages qui se sont produits entre ces 2 heures a été notée, pour les autres, elle a été estimée.

L'état, le poids et le sexc des agneaux ont été relevés.

\section{RÉSULTATS-DISCUSSION}

Sur les 224 brebis, 7 ont agnelé avant d'être traitées, soit 3, I p. 100 des animaux; ce faible pourcentage montre que la méthode de mise en lot des brebis a bien réduit les risques de parturition non induite. Neuf brebis ont reçu plus d'une injection de dexaméthasone, 208 en ont reçu une seule dont 74,5 p. Ioo les $143^{e}, 144^{e}$ et $145^{e}$ jours de gestation, 6,7 p. Ioo et I 8,7 p. Ioo le $\mathrm{I}_{4} 2^{\mathrm{e}}$ et le $\mathrm{I}_{4} 6^{\mathrm{e}}$ jour.

Le tableau I montre que les répartitions des agnelages incluits sont fonction du stade de gestation auquel les brebis ont reçu la dexaméthasone, comme nous l'avions précédemment observé (Bosc, I97I-I972). Ainsi, au cours des 24 h qui suivent l'injection, il y a moins de 2 p. Ioo de mise-bas pour les brebis traitées au $142^{\mathrm{e}}, \mathrm{I} 43^{\mathrm{e}}$ ou au $\mathrm{I}_{4} 4^{\mathrm{e}}$ jour de gestation, ces taux atteignant 4,5 p. I oo et 20,5 p. I oo pour celles qui ont reçu la dexaméthasone le $145^{\circ}$ et le $I_{4} 6^{\mathrm{e}}$ jour. Au cours des deux jours suivants (soit 24 à $72 \mathrm{~h}$ après injection) 85,7 à $92,0 \mathrm{p}$. 1 oo des agnelages ont été induits pour les brebis traitées aux I $42^{\mathrm{e}}, \mathrm{I} 43^{\mathrm{e}}, \mathrm{I} 44^{\mathrm{e}}$ et $\mathrm{I} 45^{\mathrm{e}}$ jours de gestation, contre $74,5 \mathrm{p}$. IOO pour celles qui ont été traitées au $146^{\mathrm{e}}$ jour. La majorité des mise-bas se produit donc en 2 jours, ce qui est très différent de l'aggnelage témoin pour lequel les fréquences quotidiennes de mise-bas. ont été indiquées dans le tableau $\mathrm{I}$.

La mortalité périnatale a frappé 7,5 p. Ioo des agneaux nés de toutes les brebis traitées. Le tableau 2 présente les variations des poids de naissance des agneaux simples ou jumeaux, suivant le stade de gestation auquel les mères ont reçu la dexaméthasone. L'analyse ne permet pas de 


\section{TABLEAU I}

Répartition des agnelages après induction de la parturition à différents stades de gestation chez la brebis (race Préalpes)

\begin{tabular}{|c|c|c|c|c|c|c|c|}
\hline \multirow{2}{*}{$\begin{array}{l}\text { Brebis } \\
\text { traitées à }\end{array}$} & \multirow{2}{*}{$\begin{array}{l}\text { Nombre } \\
\text { de brebis }\end{array}$} & \multicolumn{6}{|c|}{ Répartition des agnelages ( $\%)$ après le traitement } \\
\hline & & $0-24 \mathrm{~h}$ & $2 / 48 h$ & $48-72 \mathrm{~h}$ & $72-96 \mathrm{~h}$ & $96-120 \mathrm{~h}$ & $0-120 \mathrm{~h}$ \\
\hline G 142 & 14 & 0 & $\begin{array}{l}35,7 \\
(1,1)\end{array}$ & $\begin{array}{l}50,0 \\
(3,1)\end{array}$ & $\begin{array}{c}7,2 \\
(7,0)\end{array}$ & $\begin{array}{c}7,1 \\
(12,9)\end{array}$ & $\begin{array}{l}100,0 \\
(24,1)\end{array}$ \\
\hline G 143 & 39 & $\begin{array}{c}0 \\
(1,1)\end{array}$ & $\begin{array}{l}48,7 \\
(3,1)\end{array}$ & $\begin{array}{l}41,0 \\
(7,0)\end{array}$ & $\begin{array}{c}10,3 \\
(12,9)\end{array}$ & $\begin{array}{c}0,0 \\
(18,3)\end{array}$ & $\begin{array}{l}100,0 \\
(42,4)\end{array}$ \\
\hline G 144 & 50 & $\begin{array}{c}0 \\
(3,1)\end{array}$ & $\begin{array}{l}72,0 \\
(7,0)\end{array}$ & $\begin{array}{c}20,0 \\
(12,9)\end{array}$ & $\begin{array}{c}4,0 \\
(18,3)\end{array}$ & $\begin{array}{c}4,0 \\
(20,1)\end{array}$ & $\begin{array}{l}100,0 \\
(61,4)\end{array}$ \\
\hline G 145 & 66 & $\begin{array}{c}4,5 \\
(7,0)\end{array}$ & $\begin{array}{c}71,3 \\
(12,9)\end{array}$ & $\begin{array}{c}18,1 \\
(18,3)\end{array}$ & $\begin{array}{c}1,6 \\
(20,1)\end{array}$ & $\begin{array}{c}3,0 \\
(17,2)\end{array}$ & $\begin{array}{c}98,5 \\
(75,5)\end{array}$ \\
\hline G 146 & 39 & $\begin{array}{c}20,5 \\
(12,9)\end{array}$ & $\begin{array}{c}66,7 \\
(18,3)\end{array}$ & $\begin{array}{c}7,6 \\
(20,1)\end{array}$ & $\begin{array}{c}2,6 \\
(17,2)\end{array}$ & $\begin{array}{c}2,6 \\
(11,4)\end{array}$ & $\begin{array}{l}100,0 \\
(79,9)\end{array}$ \\
\hline
\end{tabular}

G $0=$ jour de saillie.

Traitement $=16 \mathrm{mg}$ de dexaméthasone.

( ) \% des mise-bas témoins calculés.

TABLEAU 2

Poids des agneaux des brebis traitées avec $16 \mathrm{mg}$ de dexaméthasone à différents stades de gestation

\begin{tabular}{|c|c|c|c|c|c|c|c|}
\hline \multirow{2}{*}{\multicolumn{2}{|c|}{ Agneaux }} & \multicolumn{6}{|c|}{ Brebis traitées } \\
\hline & & G 142 & $\mathrm{G} 143$ & G 144 & G 145 & G 146 & Témoins \\
\hline Simples & $\begin{array}{l}\text { nombre } \ldots \ldots \ldots \\
\text { poids moyen }(\mathrm{kg}) \ldots \\
\text { écart-type }(\mathrm{kg}) \ldots \ldots\end{array}$ & $\begin{array}{c}7 \\
4,11 \\
0,60\end{array}$ & $\begin{array}{c}27 \\
3,85 \\
0,48\end{array}$ & $\begin{array}{l}27 \\
4,10 \\
0,61\end{array}$ & $\begin{array}{c}40 \\
4,13 \\
0,51\end{array}$ & $\begin{array}{c}20 \\
4,06 \\
0,50\end{array}$ & $\begin{array}{c}39 \\
4,07 \\
0,49\end{array}$ \\
\hline Jumeaux & $\begin{array}{l}\text { nombre... . . . . . } \\
\text { poids moyen }(\mathrm{kg}) \ldots \\
\text { écart-type }(\mathrm{kg}) \ldots\end{array}$ & $\begin{array}{c}12 \\
3,36 \\
0,58\end{array}$ & $\begin{array}{c}21 \\
3,51 \\
0,57\end{array}$ & $\begin{array}{c}39 \\
3,44 \\
0,47\end{array}$ & $\begin{array}{c}47 \\
3,44 \\
0,54\end{array}$ & $\begin{array}{c}35 \\
3,40 \\
0,56\end{array}$ & $\begin{array}{c}22 \\
3,37 \\
0,59\end{array}$ \\
\hline
\end{tabular}

G $0=$ Jour de la saillie. 
mettre en évidence des différences significatives entre les lots ainsi constitués bien que les gestations des brebis traitées aux $\mathrm{4}^{\mathrm{e}}, \mathrm{I}_{43^{\mathrm{e}}}$ et $\mathrm{I} 44^{\mathrm{e}}$ jours de gestation aient été légèrement raccourcies.

La figure I présente le déroulement des traitements et des mise-bas comparé à un agnelage témoin calculé. Comme l'indiquent les résultats du tableau $I$, les parturitions sont groupées le $2^{\mathrm{e}}$ et le $3^{\mathrm{e}}$ jour qui suivent le traitement : il y a peu ou pas de mise-bas le jour de celui-ci, la veille

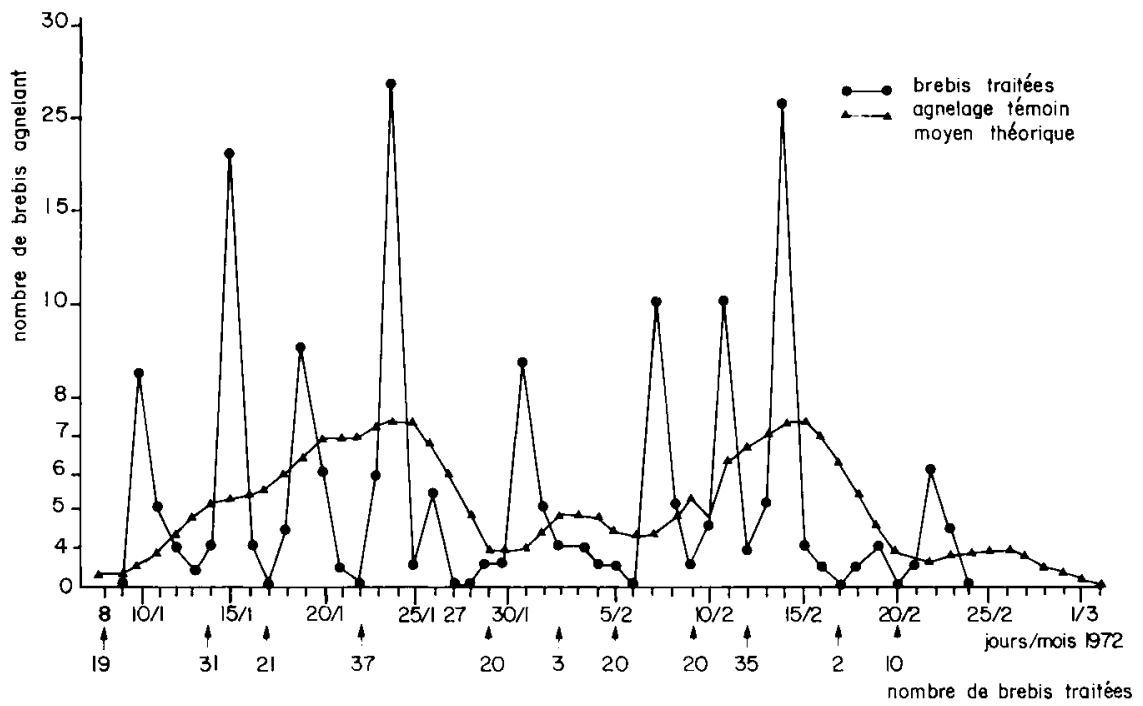

FIG. I. - Essai de groupage des agnelases après une lutte naturelle (brebis de race préalpes)

et le lendemain, Il y eut 7 jours sans agnelages et seulement 2 jours avec plus de 4 mise-bas, pour une clurée totale de 45 jours alors que l'étendue totale de l'agnelage témoin peut être estimée à 49 jours dont 23 avec plus de 4 misc-bas quotidiennes.

Pour réaliser un tel groupage cles mise-bas après une lutte naturelle, il faut évidemment connaître la date de saillic des animaux, la durée moyenne de gestation et sa variabilité, paramètres qui peuvent être aisément connus.

De plus, cette technique implique que la constitution de lots de brebis puisse être réalisée facilement, ce qui permet alors de limiter la surveillance aux animaux qui vont agneler. Cette expérience montre que le traitement ct l'organisation du troupeau qu'il implique, réduit de façon appréciable le travail du berger à ce moment.

Rę̧u pour publication en novembre 1972.

\section{SUMMARY}

\section{SYNCHRONIZATION OF I AMBINGS IN EWES AFTER NATURAL MATING} ewes.

Synchronization of lambings after natural mating was attempted in a flock of Préalpes

Dexamethasone was used to induce parturition. Every 4 or 5 days, the ewes having reached the beginning of the lambing period (i.e. 2 to 6 days before mean date for parturition) received 
an intramuscular injection of $16 \mathrm{mg}$ of this product. For each group of animals thus established lambings (about 9o p. Ioo) took place the 2nd and 3 rd day following the treatment.

This experiment shows that herd control can be better organized out that a noticeable reduction of the number of days with lambings can be obtained.

\section{RÉFÉRENCES BIBLIOGRAPHIQUES}

Adams W. M., I969. The elective induction of labor and parturition in cattle. J. Am. Vet. Med. Ass., $154,26 \mathrm{I}-265$.

Bosc M.-J., I97o. Étude de I'induction de la parturition chez la brebis par un corticoide de synthèse, le fluorométhyl prednisolone. C. R. Acad. Sci., Paris, 270, Série D, 3127-3130.

Bosc M.-J., I97I. The control of parturition in the ewe. J. Reprod. Fert., 27, 491 .

Bosc M.-J., 1972. The induction and synchronization of lambing with the aid of dexamethasone. $J$. Reprod. Fert., 28, 347-357. 